\section{Unzulässige Beteiligung von Radiologen an Kontrastmittelunternehmen und deren Rechtsfolgen - Ein unhanseatischer Fall}

\section{Einführung}

Der Bundesgerichtshof (BGH) hat mit einem Beschluss vom 25.07.2017 (Az. 5 StR 46/17) die Entscheidung des Landgerichtes Hamburg vom 18.08.2016 in einem aufsehenerregenden Strafverfahren bestätigt, das die Radiologen in Deutschland über mehrere Jahre beschäftigte. Gegenstand des Verfahrens waren nicht die im Jahr 2016 neu eingeführten Straftatbestände der Bestechlichkeit und Bestechung im Gesundheitswesen nach §299a StGB. Vielmehr betrafen die Rechtsfragen des Verfahrens die Straftatbestände des Betruges und der Untreue nach den $\S \S 263,266$ StGB. Der rechtliche Kern der Straftaten betraf die Frage nach der Zulässigkeit der Beteiligung eines Radiologen an einem Unternehmen von Leistungserbringern nach $\S 128$ Abs. 2 S. 3 und Abs. 6 Sozialgesetzbuch Fünftes Buch (SGB V), welches den Einkauf von Kontrastmitteln zum Gegenstand hatte.

\section{Abrechnungsbestimmungen für Kontrastmittel im Bereich der Kassenärztlichen Vereinigung Hamburg (KVH)}

Hintergrund der Entscheidung des BGH war der Fall eines vertragsärztlich tätigen Radiologen in Hamburg, der in den Jahren 2010 und 2012 als Ärztlicher Leiter mehrere radiologische Praxen in Norddeutschland betrieb, die überwiegend als Betriebsstätten Medizinischer Versorgungszentren (MVZ) in Form einer GmbH geführt wurden. Alleiniger Gesellschafter der $\mathrm{GmbH}$ war der Radiologe, der daneben auch als Vertragsarzt tätig war. In den Betriebsstätten wurden von angestellten Ärzten Untersuchungen mittels CT und MRT nativ oder unter Applikation von Kontrastmitteln durchgeführt. Während jener Zeit durften Kontrastmittel im Zuständigkeitsbereich der Kassenärztlichen Vereinigung Hamburg (KVH) nur als Sprechstundenbedarf und nur als Ersatz für verbrauchte Mittel zum Ende eines jeden Quartals verordnet werden, wobei die Vertragsärzte bei Verordnung, Bezug und Verwendung den Grundsatz der Wirtschaftlichkeit zu beachten hatten. Die Verordnung musste den Bedürfnissen der jeweiligen vertragsärztlichen Praxis entsprechen und zur Zahl der Behandlungsfälle in einem angemessenen Verhältnis stehen. Nach Unterschrift des Vertragsarztes und Beifügen seines Stempels konnte die Verordnung an den Lieferanten oder direkt an den Hersteller weitergereicht werden. Diese lieferten das verordnete Kontrastmittel an die Arztpraxis und rechneten ihrerseits gegenüber den Krankenkassen ab. Zwar war der Arzt verpflichtet, die Kontrastmittel möglichst günstig zu beziehen, er durfte aber den Lieferanten selbst auswählen, sofern er nicht direkt beim Hersteller bestellte. Die Lieferanten durften gegenüber Herstellern Rabatte aushandeln, ihrerseits aber den Krankenkassen gegenüber zum offiziellen Herstellerabgabepreis abrechnen und die Differenz als Gewinn verbuchen. Sinn dieser Regelung war es, eine Existenzgrundlage für den Zwischenhandel zu schaffen und zu erhalten. Eine Änderung dieser Rechtslage erfolgte in Hamburg zu Beginn des vierten Quartals 2015.

\section{Strafrechtlicher Sachverhalt der BGH-Entscheidung}

Nach den Feststellungen des BGH habe der Radiologe, um einer Zahlungsunfähigkeit zu entgehen, mit anderen Beteiligten ein Beteiligungsmodell an einem pharmazeutischen Großhändler entwickelt. So sei es zu einer sog. atypischen stillen Beteiligung an einem pharmazeutischen Großhändler seitens eines ausschließlich von dem Radiologen gehaltenen Unternehmens gekommen. Der Radiologe habe eine Einlage in Höhe von 230.000,00 Euro geleistet und erhielt $90 \%$ des Gewinns des pharmazeutischen Großhändlers. Im Zeitraum von Juli 2011 bis September 2012 kam es nach den Feststellungen des Landgerichtes Hamburg zu einer Vielzahl von Kontrastmittelverordnungen in einem Gesamtvolumen von fast

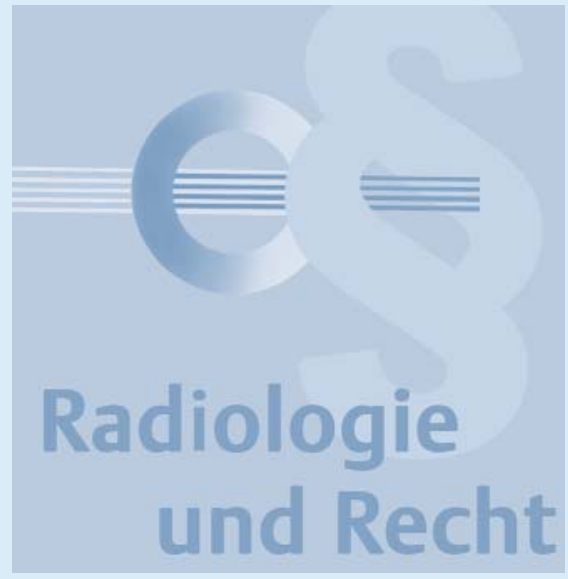

35 Millionen Euro. Sämtliche Verordnungen hätte zentral der niedergelassene Radiologe gesteuert. Der Radiologe habe seinen Mitarbeitern jeweils vorgegeben, welche Mengen des jeweiligen Kontrastmittels auf die LANR welchen Arztes in die Verordnungen einzutragen seien. Dies sei stets ohne jeden Bezug zum tatsächlichen Bedarf oder zurückliegenden Verbrauch an Kontrastmitteln erfolgt. Die Mengen seien willkürlich unter dem Gesichtspunkt des zur Aufrechterhaltung der Liquidität benötigten Gewinns aus der stillen Beteiligung festgelegt worden. Eine Vielzahl von Verordnungen habe der Radiologe auf seinen Namen unterzeichnet und darüber hinaus habe er selbst - teils mit unleserlichen Kürzeln - auf andere Ärzte ausgestellte Verordnungen unterschrieben.

Nachdem im November 2012 Verhandlungen mit misstrauisch gewordenen Krankenkassen gescheitert seien und erhebliche Regressforderungen gedroht hätten, folgten die Insolvenzen der MVZ des Vertragsarztes. Der Radiologe habe sich ins Ausland abgesetzt und entziehe sich nach den Feststellungen des BGH seitdem der Strafverfolgung. Das Landgericht Hamburg verurteilte den Geschäftsführer des pharmazeutischen Großhändlers wegen Betruges und Beihilfe zum Betrug zu einer Gesamtfreiheitsstrafe von fünf Jahren und den Geschäftsführer der MVZ wegen Beihilfe zum Betrug zu einer Freiheitsstrafe von vier Jahren und sechs Monaten.

Aus dem Beschluss des BGH gehen mehrere Feststellungen hervor, die insbesondere die unmittelbare und mittelbare Beteiligung von Vertragsärzten an Unternehmern von 
Leistungserbringern betreffen. Unternehmen von Leistungserbringern können nach § 128 Abs. 6 S. 1 SGB V z. B. Apotheken, pharmazeutische Großhändler, pharmazeutische Unternehmen oder sonstige Anbieter von Gesundheitsleistungen sein.

\section{Stillschweigende Erklärung gegenüber den Krankenkassen durch die Einreichung von Verordnungen}

Die Rechtsprechung der Sozialgerichte und der Strafgerichte betraf in der Vergangenheit häufig die Bedeutung der quartalsweisen Sammelabrechnung des Vertragsarztes gegenüber der Kassenärztlichen Vereinigung. Beide Gerichtszweige verstehen die Sammelerklärung als Garantieerklärung. Die Sammelerklärung stellt insoweit eine Garantie dar, die der unterzeichnende Vertragsarzt z. B. hinsichtlich der Vollständigkeit der Erbringung des obligaten und fakultativen Leistungsinhaltes der Gebührenordnungspositionen, der persönlichen Leistungserbringung, der Voraussetzungen etwaiger Vertretertätigkeiten oder Genehmigung von Angestellten, dem Vorliegen und Fortbestand von Abrechnungsgenehmigungen aufgrund einer Qualitätssicherungsvereinbarung nach $\S 135$ Abs. 2 SGB $\checkmark$ und hinsichtlich der Einhaltung anderer vertragsärztlicher Regelungen abgibt. Nicht jeder radiologische Vertragsarzt misst der Sammelerklärung einen solchen, weitgehenden Inhalt bei. Ausdrücklich geregelt ist die Verordnung von gesondert berechnungsfähigen Materialien nach $\S 44$ Abs. 6 Bundesmantelvertrag-Ärzte (BMV-Ä). Dabei bestimmt §44 Abs. 6 S. 7 BMV-Ä, dass der Vertragsarzt verpflichtet ist, die tatsächlich realisierten Preise in Rechnung zu stellen und ggf. vom Hersteller bzw. Lieferanten gewährte Rückvergütungen, wie Preisnachlässe, Rabatte, Umsatzbeteiligungen, Bonifikationen und rückvergütungsgleiche Gewinnbeteiligungen mit Ausnahme von Barzahlungsrabatten bis zu 3 \% weiterzugeben. Der Vertragsarzt bestätigt dies durch Unterschrift gegenüber der rechnungsbegleichenden Stelle.
In dem Verfahren vor dem BGH ging es nicht um die Sammelerklärung des Radiologen, sondern um die Abrechnung eines pharmazeutischen Großhändlers als Leistungserbringer gegenüber den gesetzlichen Krankenkassen. Ähnlich, wie bei der Sammelerklärung, stellte der BGH auf die durch die Vorschriften des SGB V geprägten normativen Erwartungen der jeweiligen Krankenkassenmitarbeiter ab. Danach enthalte die Einreichung von Verordnungen in Zusammenhang mit entsprechenden Rechnungen regelmäßig die stillschweigende Erklärung, diese seien in geltend gemachter Höhe endgültig angefallen und nicht $z$. B. durch Kick-Back-Zahlungen an den verordnenden Arzt geschmälert (vgl. BGH, Beschluss vom 27.04.2004, Az. 1 StR 165/03). Ohne diese Feststellung seitens des $\mathrm{BGH}$ hätte es an dem Tatbestandsmerkmal „Täuschung“ eines Betruges gefehlt. Hätten die Krankenkassenmitarbeiter keine generelle Erwartung an die Abrechnung oder eine bestimmte Erwartung in dem konkreten Einzelfall, wäre eine Täuschung der Krankenkasse nicht möglich gewesen, so dass ein Betrug nicht in Betracht gekommen wäre.

\section{Beteiligungen an Unternehmen von \\ Leistungserbringern nach $\S 128$ Abs. 2 und 6 SGB V}

In dem Strafverfahren war der niedergelassene Radiologe gesellschaftsrechtlich mittelbar an einem pharmazeutischen Großhändler beteiligt. Nach $\S 128$ Abs. 2 S. 1 und Abs. 6 SGB V dürfen pharmazeutische Großhändler Vertragsärzte jedoch nicht gegen Entgelt oder Gewährung sonstiger wirtschaftlicher Vorteile an der Verordnung von Arzneimitteln - etwa Kontrastmitteln beteiligen oder solche Zuwendungen im Zusammenhang mit der Verordnung gewähren. § 128 Abs. 2 SGB V lautet auszugsweise (Satz 1 und 3):

„Leistungserbringer dürfen Vertragsärzte sowie Ärzte in Krankenhäusern und anderen medizinischen Einrichtungen nicht gegen Entgelt oder Gewährung sonstiger wirtschaftlicher Vorteile an der Durchführung der Versorgung mit Hilfsmitteln beteiligen oder solche Zuwendungen im Zusammenhang mit der Verordnung von Hilfsmitteln gewähren. [...]
Unzulässige Zuwendungen im Sinne des Satzes 1 sind auch die unentgeltliche oder verbilligte Überlassung von Geräten und Materialien und Durchführung von Schulungsmaßnahmen, die Gestellung von Räumlichkeiten oder Personal oder die Beteiligung an den Kosten hierfür sowie Einkünfte aus Beteiligungen an Unternehmen von Leistungserbringern, die Vertragsärzte durch ihr Verordnungs- oder Zuweisungsverhalten selbst maßgeblich beeinflussen."

§ 128 Abs. 6 S. 1 SGB V stellt sodann klar, dass der Anwendungsbereich des Absatz 2 sich auf pharmazeutische Großhändler, pharmazeutische Unternehmen und andere erstreckt. Die Regelung lautet:

„Ist gesetzlich nichts anderes bestimmt, gelten bei der Erbringung von Leistungen nach den $\S \S 31$ und 116b Absatz 7 die Absätze 1 bis 3 sowohl zwischen pharmazeutischen Unternehmern, Apotheken, pharmazeutischen Großhändlern und sonstigen Anbietern von Gesundheitsleistungen als auch jeweils gegenüber Vertragsärzten, Ärzten in Krankenhäusern und Krankenhausträgern entsprechend."

Um eine unzulässige Zuwendung handelt es sich nach § 128 Abs. 2 S. 3 SGB V, soweit Einkünfte aus Beteiligungen an Unternehmen von Leistungserbringern erzielt werden, die Vertragsärzte durch ihr Verordnungs- oder Zuweisungsverhalten selbst maßgeblich beeinflussen. Die Vorschrift des $\S 128$ Abs. 2 S. 3 SGB V trat bereits zum 01.01.2012 in Kraft. Nach der Gesetzesbegründung war dies lediglich eine Klarstellung des schon vorher geltenden Verbots unzulässiger Zuwendungen nach § 128 Abs. 2 S. 1 SGB a.F. (vgl. BT-Drucks. 17/6906 S. 85). Um „sonstige wirtschaftliche Vorteile“ i. S.v. §128 Abs. 2 S. 1 SGB V handelte es sich also auch schon vor dem 01.01.2012, wenn einem Arzt für sein Verordnungsverhalten Gewinne oder sonstige Einnahmen aus einer gesellschaftsrechtlichen Beteiligung zuflossen (vgl. BGH, Urteil vom 13.01.2011, Az. I ZR 111/08). Dies alles gilt nicht nur für unmittelbar dem Arzt gewährte finanzielle Vorteile, sondern auch für solche, die zunächst einer juristischen Person zufließen, mittelbar aber in erheblichem Umfang dem Vertragsarzt in seiner Eigenschaft als Gesellschafter oder Aktionär zukommen. Der „Umweg“ einer Vorteilsgewährung über eine juristische Person 
ändert nichts an der Bewertung dem Arzt zufließender geldwerter Vorteile, insbesondere, wenn es sich um einen Alleingesellschafter oder Alleinaktionär der unmittelbar begünstigten Gesellschaft handelt. Im Zusammenhang mit dem entsprechenden berufsrechtlichen Verbot für Ärzte, derartige Beteiligungen einzugehen (vgl. auch $\S 34$ MBO-Ä a.F., nunmehr §32 Abs. 1 MBO-Ä), hat der BGH vor Inkrafttreten von $\S 128$ Abs. 2 S. 3 SGB $V$ eine differenzierte Betrachtung lediglich in denjenigen Fällen für angebracht gehalten, in denen sich der Arzt nur mittelbar, insbesondere über allgemeine Gewinnausschüttungen, am Erfolg eines Unternehmens beteiligt.

Voraussetzung für eine unbedenkliche Beteiligung ist in solchen Fällen, dass bei objektiver Betrachtung ein spürbarer Einfluss des Zuweisungs- oder Verordnungsverhaltens des einzelnen Arztes auf seinen Ertrag aus der Beteiligung ausgeschlossen erscheint. Ob dies der Fall ist, hängt grundsätzlich vom Gesamtumsatz des Unternehmens, dem Anteil der Überweisungen bzw. Verordnungen des Arztes und der Höhe seiner Beteiligung ab. Die Unzulässigkeit der Beteiligung wird sich dabei schon aus der Gesamthöhe der dem Arzt aus ihr zufließenden Vorteile ergeben können, sofern diese in spürbarer Weise von seinem eigenen Verordnungs- oder Zuweisungsverhalten beeinflusst wird. Dabei kam es nach dem BGH nicht darauf an, ob der Radiologe die Verordnungen selbst ausstellte oder ob er diese als Leiter eines MVZ den dort tätigen Ärzten zur Unterschrift vorlegte und von ihnen unterzeichnen ließ.

\section{Rechtsfolge einer unzulässigen \\ Unternehmensbeteiligung}

Ein Verstoß gegen § 128 Abs. 2 und 6 SGB V führt dazu, dass der Leistungserbringer, in dem Fall des BGH war dies nicht der Vertragsarzt, sondern der pharmazeutische Großhändler, seinen Zahlungsanspruch auf Erstattung des Herstellerabgabepreises für die an den Radiologen gelieferten Kontrastmittel insgesamt verlor. Nach der Rechtsprechung des Bundessozialgerichts (BSG) können Leistungserbringer für Leistungen, die sie unter Verstoß gegen die rechtlichen
Vorgaben des SGB V erbracht haben, in aller Regel keine Vergütung beanspruchen (vgl. BSG, Urteil vom 02.07.2013, Az. B 1 KR 49/12 R). Dies gilt nach dem Schutzzweck der Norm auch bei Verstößen gegen $\S 128$ Abs. 2 und 6 SGB V, denn diese Vorschrift soll Versicherte und die Krankenkassen vor einem kollusiven Zusammenwirken von Ärzten und Leistungserbringern zu ihrem Nachteil schützen (vgl. zur Gesetzesbegründung BT-Drucks. 16/10609 S. 58; zur „streng formalen Betrachtungsweise“ beim Abrechnungsbetrug $\mathrm{BGH}$, Beschluss vom 16.06.2014, Az. 4 StR 21/14). Seinen Vergütungsanspruch gegenüber der Krankenkasse habe der Leistungserbringer in dem hier vorliegenden Sachverhalt, nach Ansicht des $\mathrm{BGH}$, durch den Verstoß gegen das gesetzliche Verbot des $\S 128$ Abs. 2 und 6 SGB $\vee$ aufgrund der - verheimlichten - Kick-back-Zahlungen an den Radiologen verloren. Die weitere Folge des Verstoßes gegen das gesetzliche Verbot ist, dass der Radiologe seinen Anspruch auf Beteiligung nicht gerichtlich durchsetzen könnte, wenn der Geschäftsführer des pharmazeutischen Großhändlers die Gewinne nicht abgeführt hätte. Das Recht hilft danach denjenigen nicht, die sich außerhalb des Rechtes stellen.

\section{Vermögensbetreuungs- pflichten}

Den Vertragsarzt selbst trifft aufgrund seiner besonderen Befugnisse (sog. Schlüsselfigur) bei seiner Verordnungstätigkeit grundsätzlich eine Vermögensbetreuungspflicht zugunsten der gesetzlichen Krankenkassen (vgl. ausführlich hierzu BGH, Beschluss vom 16.08.2016, Az. 4 StR 163/ 16). Eine Untreue setzt $u$.a. voraus, dass der Täter eine Vermögensbetreuungspflicht gegenüber demjenigen haben muss, dessen Vermögen Nachteile erfährt. Dies gilt auch bei der Verordnung von Sprechstundenbedarf, denn der verordnende Radiologe hat es insoweit in der Hand, die gesetzlichen Krankenkassen zu entsprechenden Zahlungen zu verpflichten, ohne dass diesen eine hinreichende Kontrollmöglichkeit zur Verfügung steht. Der BGH beurteilte das Verhalten des Radiologen als eine Untreue durch Verletzung der Vermögensbetreuungspflicht auch in denjenigen Fällen, in denen er nicht selbst die Verord- nungen unterschrieb, sondern gutgläubigen Ärzten die vorausgefüllten Verordnungsvordrucke zur Unterschrift vorgelegt habe, um sie anschließend über den pharmazeutischen Großhändler bei den gesetzlichen Krankenkassen mit dem Ziel einreichen zu lassen, von den dadurch erwirtschafteten Gewinnen selbst zu profitieren. Dadurch habe der Radiologe letztlich den gesamten Verordnungsvorgang für Kontrastmittel in allen Bereichen seines Konzerns unmittelbar selbst gesteuert.

\section{Fazit}

Die klare Erkenntnis aus diesem Verfahren ist, dass niedergelassene Ärzte bei der Beteiligung an Unternehmen von Leistungserbringern, mit denen sie im Rahmen ihrer ärztlichen Tätigkeit in einer Geschäftsbeziehung stehen, äußerste Zurückhaltung üben sollten. Dies gilt insbesondere dann, wenn der Arzt durch sein Zuweisungsverhalten den Gewinn des Unternehmens und damit zugleich seinen eigenen wirtschaftlich Vorteil positiv beeinflussen kann. Dieses Gebot gilt nicht nur für den Vertragsarzt, sondern für jeden Arzt, da ein solches Verhalten zugleich gegen das Verbot der Vorteilsannahme i.S.d. §31 Muster-Berufsordnung (MBO-Ä) verstößt.

Diese Feststellungen hat das OLG Stuttgart bereits in einer Entscheidung vom 10.05.2007 (Az. 2 U 176/06 - vgl. RöFo, 2008, S. 933 ff.) und ihm folgend das Landesberufsgericht für Heilberufe Münster mit Urteil vom 06.07.2011 (Az. 6t A 1816/09.T - vgl. RöFo, 2012, S. 273 ff.) getroffen und sich insoweit eindeutig geäußert:

„Ist beim Erwerb von börsennotierten Aktien eines Pharmaunternehmens und der gezielten Verordnung gerade von Medikamenten aus diesem Hause die Selbstbelohnung äußerst mittelbar, weil die Börsenbewertung des Unternehmens von vielfältigen anderen Faktoren, welche der Arzt nicht beeinflussen kann, abhängt, weshalb diese Selbstbindung im Zuweisungsverhalten einen sehr ungewissen Lohn verspricht, ist die vorliegende Ausrichtung des Zuweisungsverhaltens auf die Gesellschaft, an der der Arzt beteiligt ist, unmittelbar werthaltig und, wenn alle Unterbeteiligten das Geschäftsmodell verinnerlicht haben und danach handeln, annähernd linear 
zum Zuweisungsgrad lukrativ. Dieses Selbstbelohnungssystem, welches von der Teilhabe des Zuweisenden am Liquidationserlös des Laborleistungen Erbringenden lebt, läuft jedoch dem Grundgebot einer nicht von Eigennutz überlagerten ärztlichen Entscheidung grob zuwider und verzerrt, kommt es zu solchen Gesellschaften, das Nachfrageverhalten nachhaltig zu Lasten von qualitativ gleichwertigen, unter Umständen gar besseren Labors, die nur eben keine Selbstprovisionierungsmöglichkeit durch das Zuweisungsverhalten eröffnen. “

Alle Versuche, durch eine komplexe gesellschaftsrechtliche Struktur Zahlungsflüsse intransparent zu gestalten, sind auch in dem vorliegenden Fall gescheitert und führten in dem Verfahren vor dem BGH gleichwohl zu einer persönlichen strafrechtlichen Zurechnung zulasten des Radiologen und des Geschäftsführers des pharmazeutischen Großhändlers. Es ist daneben eine trügerische Vorstellung, dass ein Strafgericht einen Vertragsarzt nicht als Teilnehmer an einer Straftat eines pharmazeutischen Großhändlers gegenüber den gesetzlichen Krankenkassen verurteilen könnte, wenn der pharmazeutische Großhändler den Vertragsarzt an den von ihm generierten Umsätzen beteiligt. Dem Ra- diologen gelang es nach Auffassung des $\mathrm{BGH}$ weder, das Verbot unzulässiger Unternehmensbeteiligungen nach $\S 128$ Abs. 2 S. 3 und Abs. 6 SGB V zu umgehen, in dem sich dieser nicht unmittelbar selbst an dem pharmazeutischen Großhändler beteiligte, sondern über die Zwischenschaltung einer weiteren Gesellschaft, deren Alleingesellschafter der Radiologe war. Noch führte sein Verhalten, als Ärztlicher Leiter der MVZ nicht selbst die Arzneimittelverordnungen auszustellen, sondern die Verordnungen durch angestellte, gutgläubige Ärzte unterzeichnen zu lassen, zu einer Strafbefreiung. Der Beschluss des BGH dürfte nunmehr den letzten Zweifel ausgeräumt haben, dass durch eine komplexe gesellschaftsrechtliche Struktur und verschleierte Zahlungsflüsse das gesetzliche Verbot der unzulässigen Unternehmensbeteiligung sanktionsfrei zu umgehen ist.

Daneben wird ein weiterer Aspekt in dem Beschluss des BGH deutlich. Sowohl der $\mathrm{BGH}$ als auch das BSG setzen generell voraus, dass die abstrakte Erwartung von Mitarbeitern der Krankenkassen und der Kassenärztlichen Vereinigungen an die Abrechnung oder Leistungsanforderung von Vertragsärzten ist, dass diese mit der
Abrechnung oder Leistungsanforderung erklären, sich an die vertragsärztlichen und sonstige Regelungen gehalten zu haben. Weiß der Vertragsarzt, dass die Voraussetzungen an eine regelhafte Abrechnung aufgrund von Verstößen gegen das Vertragsarztrecht nicht vorliegen und reicht dieser dennoch die Leistungen zur Abrechnung ein, liegt die Annahme eines versuchten Betruges nahe. Leistet die Kassenärztliche Vereinigung auf diese Leistungsanforderung oder begleicht die Krankenkasse die Rechnung des Vertragsarztes, liegt regelmäßig ein vollendetes Betrugsdelikt vor.

René T. Steinhäuser

Rechtsanwalt

Prof. Dr. Peter Wigge

Rechtsanwalt

Fachanwalt für Medizinrecht

Rechtsanwälte Wigge

Großer Burstah 42

20457 Hamburg

Telefon: (040) 3398705 - 90

Telefax: (040) 3398705 - 99

Internet: www.ra-wigge.de

E-Mail: kanzlei@ra-wigge.de 